https://doi.org/10.18778/2196-8403.2020.08

HEIKE KRÖSCHE

\title{
Aktuelle Entwicklungen in der Vermittlung von österreichischen Erinnerungskulturen in Schule und Unterricht
}

Im Mittelpunkt des Beitrags stehen geschichtsdidaktische Überlegungen zum aktuellen Stellenwert von Erinnerungskulturen als Gegenstand historisch-politischer Lernprozesse in der österreichischen Sekundarstufe. Zu diesem Zweck wird eine erste Analyse auf drei Ebenen durchgeführt. Zunächst wird die Relevanz des Themas Erinnerungskulturen im aktuellen Lehrplan für das Unterrichtsfach „Geschichte und Sozialkunde / Politische Bildung" betrachtet. Ergänzend dazu wird ein exemplarischer Blick auf die Umsetzung dieser Vorgabe in einem aktuellen Schulbuch geworfen. Abschließend werden dazu erste empirische Ergebnisse zu den Vorstellungen von Schülerinnen und Schülern zum Umgang mit Erinnerungskulturen in Beziehung gesetzt.

Schlüsselwörter: Gedenken, Geschichtsdidaktik, österreichische Erinnerungskulturen, Lehrerausbildung

Current developments in the communication of Austrian remembrance cultures in schools and lessons

The contribution focuses on historical-didactic considerations on the current significance of memory cultures as an object of historical-political learning processes in Austrian secondary schools. For this purpose, an initial analysis is carried out on three levels. First, the relevance of the topic of memory cultures in the current curriculum for the teaching subject "History and Social Studies / Political Education" is considered. In addition, an exemplary look is taken into the implementation of this issue in a current textbook. Finally, initial empirical results are presented on students' ideas about how to deal with memory cultures in relation to each other.

Keywords: commemorate, history pedagogy, Austrian memory culture, teacher training 
Kształcenie w zakresie kultury pamięci w austriackich szkołach - stan aktualny Artykuł zajmuje się aktualnym stanem historyczno-politycznego kształcenia w zakresie kultury pamięci w austriackich szkołach ponadpodstawowych. Analiza została przeprowadzona po raz pierwszy na trzech poziomach. Na początku artykuł stawia pytanie o znaczenie tematu, jakim jest kultura pamięci w obecnym programie przedmiotu „Historia i nauka o społeczeństwie / wiedza o polityce”. Uzupełnieniem jest spojrzenie na realizację tego zagadnienia w wybranym aktualnym podręczniku. Na koniec rozważania na temat programu kształcenia są skonfrontowane z pierwszymi empirycznymi wynikami badań, w których uczniowie pytani są o ich doświadczenia z lekcji na temat kultury pamięci.

Słowa klucze: pamięć, dydaktyka historii, austriackie kultury pamięci, kształcenie nauczycieli

\section{Einleitung: Das Gedenk- und Erinnerungsjahr 2018}

Mit dem Jahreswechsel 2018-2019 ging in Österreich das „Gedenk- und Erinnerungsjahr 2018“ zu Ende - ein Ereignis, das nicht nur Einblick in die geschichtspolitische, sondern auch die geschichtskulturelle Dimension des Umgangs mit der Vergangenheit gibt. Dabei sind Gedenkjahre oder Jubiläen nach Klaus Bergmann von jährlich wiederkehrenden Gedenktagen wie Nationaloder Staatsfeiertagen zu unterscheiden. Anlass ist ein relevantes Ereignis, das sich nach einer runden Jahreszahl oder bestimmten Zahlenkombination wiederholt (vgl. BERGMANN 2009:25f.). 2018 war jedoch ein Gedenkjahr, das gleich auf mehrere Jahreszahlen und damit mehrere historische Bezugspunkte rekurrierte. Derartige Jahre des Gedenkens haben in Österreich seit 1988 eine gewisse Tradition ${ }^{1}$ und erreichten 2018 einen vorläufigen Höhepunkt.

Nicht nur thematisch wurde vom 100. Jahrestag des Endes des Ersten Weltkrieges bis zum 50. Jahrestag des Prager Frühlings ein großer Bogen des Gedenkens sowohl mit negativen als auch positiven Referenzpunkten des Erinnerns gespannt, auch das Spektrum der angebotenen Formate war mit offiziellen Gedenkveranstaltungen, Vorträgen, Diskussionen, Publikationen und schließlich der medienwirksamen Eröffnung des Hauses der Geschichte Österreich in der Neuen Burg Wien breit. ${ }^{2}$ Im Zuge dieses Erinnerungs- und Gedenkjahres ist einerseits

Zum Gedenkjahr 1988 vgl. KNIGHT 2006.

2 Ein Überblick über die verschiedenen Formate findet sich in der Event- und Publikationsdatenbank der offiziellen Homepage zum Gedenk- und Erinnerungsjahr 2018. https://www.oesterreich100.at/events-und-publikationen.html (30.09.2019) 
die Bereitschaft zu kritischen Tönen deutlich geworden, etwa als der Bundespräsident Alexander van der Bellen sich im Rahmen des Staatsaktes am 12. März 2018 gegen jegliches Vergessen und für eine Stärkung der Demokratie aussprach und zugleich Österreichs Mitverantwortung für die Gräueltaten des Nationalsozialismus betonte (vgl. GERALD 2018). Andererseits ist der Mehrwert dieses Überangebots insbesondere in Hinblick auf die zentralen Erinnerungsfunktionen (vgl. ASSMANN 2005) mehr als fraglich und es besteht die Gefahr einer Übersättigung gerade durch die öffentliche und mediale Dauerpräsenz (vgl. WELSKOPP 2014).

Diese Ambivalenz stellt auch eine Herausforderung für den Unterricht dar, sodass der Schule als Vermittlungsinstanz eine große Verantwortung zukommt (vgl. UHL 2010:13). Die Notwendigkeit, Erinnerungskulturen zum Gegenstand historisch-politischen Lernens zu machen, steht dabei außer Frage. Ein veränderter Zugang zu einer ,Didaktik des Erinnerns' ist in Österreich jedoch erst mit Beginn des 21. Jahrhunderts intensiv diskutiert worden (vgl. KRAMMER 2003). Der historisch-politische Unterricht hat also die Aufgabe, nicht einzelne historische Ereignisse als Bezugspunkte des Gedenkens, sondern den Umgang mit der Vergangenheit in Form von Erinnerungskulturen im Wandel der Zeit einschließlich seiner gesellschaftspolitischen Funktionen zum Gegenstand des Lernens zu machen und dafür einen kritisch-analytischen Zugang zu wählen (vgl. KÜHBERGER 2010).

Vor diesem Hintergrund soll im Folgenden der Blick auf den Stellenwert österreichischer Erinnerungskulturen in Schule und Unterricht gerichtet werden, womit geschichtsdidaktische Überlegungen in den Mittelpunkt rücken. Ziel ist eine aktuelle Bestandsaufnahme, die Voraussetzung ist, um in weiterer Folge Implikationen für den Unterricht und damit auch die Lehrerinnen- und Lehrerausbildung abzuleiten. Zu diesem Zweck findet zunächst eine Auseinandersetzung mit dem Begriff ,Erinnerungskulturen“ und der schon seit einigen Jahrzehnten diskutierten Frage der ,Zukunft der Erinnerung“ (KNIGGE 2010) statt. Im Anschluss daran wird die Relevanz des Themas Erinnerungskulturen für historisch-politische Lernprozesse anhand von drei Zugängen diskutiert: dem jeweils aktuellen Stellenwert in den Lehrplänen und Schulbüchern für die Sekundarstufe I und im Spiegel von bisherigen empirischen Erkenntnissen. Damit will der Beitrag Impulse für die weitere Beschäftigung mit dem Thema sowohl in empirischer als auch in pragmatischer Hinsicht liefern. 


\section{Erinnerungskulturen und die Kontroverse um die „Zukunft der Erinnerung“"}

Zur Analyse des öffentlichen Umgangs mit Vergangenheit haben sich im deutschsprachigen Raum unterschiedliche Konzepte entwickelt, ${ }^{3}$ wovon für diesen Beitrag das Konzept der Erinnerungskulturen zentral ist. Dieses lässt sich jedoch nicht losgelöst vom Paradigma des kollektiven (bzw. kulturellen) Gedächtnisses betrachten, denn ,erinnert werden kann nur, was sich im Gedächtnis befindet" (HASBERG 2004:199). Dementsprechend haben sich sowohl ,Gedächtnis‘ als auch ,Erinnerung' als Leitbegriffe im kulturhistorischen Diskurs bewährt, bevor Erinnerungskulturen im Zuge der 1990er-Jahre zum geschichtswissenschaftlichen und vor allem zeitgeschichtlichen Forschungskonzept avancierten (vgl. CORNELIßEN 2012).

Zur Begriffsgeschichte gehört die Kontroverse, ob der Vorgang des Erinnerns individuell oder kollektiv getragen wird (vgl. HASBERG 2004:200f.), was aber hier nicht im Detail aufgegriffen werden soll. Stattdessen bietet sich für das Anliegen, Erinnerungskulturen zum Gegenstand fachübergreifender Lehr- und Lernprozesse zu machen, ein weites Begriffsverständnis nach Cornelißen an. Für ihn erscheint es sinnvoll, „,Erinnerungskultur“ als einen formalen Oberbegriff für alle denkbaren Formen der bewussten Erinnerung an historische Ereignisse, Persönlichkeiten und Prozesse zu verstehen, seien sie ästhetischer, politischer oder kognitiver Natur" (CORNELIßEN 2012:2). Dementsprechend würden ,als Träger dieser Kultur [...] Individuen, soziale Gruppen oder sogar Nationen in Erscheinung [treten], teilweise in Übereinstimmung miteinander, teilweise aber auch in einem konfliktreichen Gegeneinander" (CORNELIßEN 2012:2). Die so verstandenen Erinnerungsprozesse im ,Spannungsfeld zwischen subjektiver Erfahrung, wissenschaftlich objektivierter Geschichte und kultureller Kommemoration“ (CORNELIßEN / KLINKHAMMER / SCHWENTKER 2003:12) sind gerade aufgrund des heterogenen Zugangs auch für den geschichtsdidaktischen Diskurs interessant.

So beschreibt Astrid Erll Erinnerungskulturen als die „historisch und kulturell variablen Ausprägungen von kollektivem Gedächtnis“ (ERLL 2008:176). Die Pluralform zeige zudem, dass ,wir es niemals, auch nicht in den homogensten Kulturen, mit nur einer einzigen Erinnerungsgemeinschaft zu tun haben" (ERLL 2008:176), weshalb auch in diesem Beitrag ausschließlich diese Form verwendet wird. Der Akzent auf Erinnerung wiederum signalisiere, dass „,das

3 Vgl. den Überblick bei Demantowsky (2015). 
wissenschaftliche Konstrukt ,kollektives Gedächtnis' erst in seiner Aktualisierung durch einzelne kollektive Erinnerungsakte tatsächlich beobachtbar und kulturwissenschaftlich analysierbar wird“" (ERLL 2008:176). Diese Feststellung gilt nicht nur gleichermaßen für die Geschichtswissenschaft, sondern schlägt auch eine Brücke zu dem zentralen geschichtsdidaktischen Prinzip der Gegenwartsund Zukunftsorientierung.

In den vergangenen Jahren wurde der Erinnerungsdiskurs zudem von der Frage nach der ,Zukunft der Erinnerung' geprägt, die nicht zuletzt vor dem Hintergrund des generationellen Umbruchs bzw. in Hinblick auf die Holocaust-Überlebenden des sogenannten „lange[n] Ende[s] der Zeitzeugenschaft“ (TAUBITZ 2016) aufgebrochen ist. Eine Verlängerung der „Ära der Zeitzeugen“ (TAUBITZ 2016:14) durch videografierte Interviews löst in der Holocaust-Vermittlung die reale Begegnung mit Zeitzeugen durch eine virtuelle ab und hat Auswirkungen auf den Lernprozess. ${ }^{4}$ Für die in diesem Zusammenhang vor allem in Deutschland ausgetragene Kontroverse um das ,Unbehagen an der Erinnerungskultur ${ }^{6}$ kommt als weiterer Aspekt hinzu, dass sich inzwischen Kritiker und Kritikerinnen aufgrund der zunehmenden medialen Präsenz und Kommerzialisierung von Erinnerung von diesem Trend distanzieren und abwertend von „Erinnerungsindustrie“ oder „Erinnerungstourismus“ sprechen (WOLFRUM 2011:209). Die Intervention von Aleida Assmann gegen eine Abschaffung der Erinnerungskultur(en) und für mehr begriffliche Differenzierung (vgl. ASSMANN 2013) scheint nicht nur angemessen, sondern kann auch für einen kritisch-analytischen Zugang im Geschichtsunterricht fruchtbar gemacht werden.

\section{Vermittlung österreichischer Erinnerungskulturen in der historisch- politischen Bildung}

\subsection{Erinnerungskulturen als Gegenstand des österreichischen Lehrplans für Geschichte und Sozialkunde / Politische Bildung}

Dem aktuellen Lehrplan für das Fach Geschichte und Sozialkunde / Politische Bildung der Neuen Mittelschule und der AHS-Unterstufe, der auf einer Synthese von Geschichts- und Politikunterricht beruht und folgerichtig Erinnerungskulturen als Gegenstand des historisch-politischen Lernens fest verankert, liegt ein längerer Entwicklungsprozess zugrunde. Für diese Genese ist der Einfluss des

4 Siehe dazu die empirische Studie zum Einsatz videografierter Zeitzeugeninterviews im Unterricht von CHRISTINA ISABEL BRÜNING (2018). 
politisch-gesellschaftlichen Umgangs mit der Zeit des Nationalsozialismus in Österreich auf den Geschichtsunterricht genauso entscheidend wie die geschichtswissenschaftliche Aufarbeitung.

Christoph Kühberger und Herbert Neureiter haben dementsprechend für den Umgang mit Nationalsozialismus und Holocaust aus geschichtsdidaktischer Perspektive vier Phasen unterschieden: 1. die Phase der Nicht-Thematisierung ab 1945, 2. parallel dazu ab 1945 die Phase der Positionierung der Opferthese, 3. ab der Waldheim-Affäre 1986 die Positionierung der Täter/innen-Opfer-These und 4. mit dem beginnenden 21. Jahrhundert eine einsetzende Pluralisierung der Gedenk- und Erinnerungskultur (vgl. KÜHBERGER / NEUREITER 2017:21). Diesen Phasen entsprechend lag das Hauptaugenmerk des Geschichtsunterrichts bis in die Mitte der 1980er Jahre auf dem Zweiten Weltkrieg (vgl. MITTNIK 2018:113). Darin spiegelt sich der offizielle Umgang mit dem Thema NS-Vergangenheit wider, das man laut Hammerstein in den frühen 1980er Jahren in Österreich „im Grunde für ,erledigt“ erachtete“ (HAMMERSTEIN 2017:189).

Dem Narrativ des ersten Opfers des Nationalsozialismus folgend wurde im Unterricht zudem vermittelt, dass die Mehrheit der österreichischen Bevölkerung dem Nationalsozialismus distanziert gegenübergestanden hätte (vgl. KRAMMER 2003:14). Eine erste Zäsur in diesem Selbstverständnis markierte die WaldheimAffäre im Jahre 1986, die auch einen wichtigen geschichtsdidaktischen Impuls auslöste, in dessen Folge das Thema der österreichischen Mitverantwortung für die nationalsozialistischen Gewaltverbrechen Eingang in den österreichischen Geschichtsunterricht fand (vgl. MITTNIK 2018:113). Dennoch hat der österreichische Geschichtsdidaktiker Reinhard Krammer noch 2003 die auf Betroffenheit ausgerichtete Didaktik des Erinnerns in Österreich kritisiert (vgl. KRAMMER 2003:14-16). Problematisch stellte sich für ihn dar, dass die Auseinandersetzung mit der österreichischen Verantwortung im Unterricht nur halbherzig stattfinden würde. Statt ein reflektiertes Geschichtsbewusstsein anzubahnen, stünden Rechtfertigungsstrategien und eine Reduzierung von Verantwortung auf die Person Hitlers und seine engsten Mitarbeiterinnen und Mitarbeiter im Mittelpunkt (vgl. KRAMMER 2003:15f.). Durch diese verengte Perspektive könne, so Krammers Kritik, auch kein „Verständnis für die Notwendigkeit, sich an die Ereignisse zu erinnern" entwickelt werden (KRAMMER 2004:3).

Mit seiner kritischen Einschätzung hat Reinhard Krammer entscheidend zum Diskurs um neue fachdidaktische Konzepte beigetragen. Ein Ergebnis ist, dass „Erinnerungslernen“ heute durch historisch-politische Bildung geprägt ist (Hellmuth 2018). Bezogen auf die Phasen des Umgangs mit Nationalsozialismus und Holocaust nach Kühberger und Neureiter befindet sich Österreich 
also seit der Jahrtausendwende in der Phase der Pluralisierung der Gedenkund Erinnerungskultur, zu deren didaktischen Kennzeichen dem Paradigmenwechsel entsprechend die Kompetenzorientierung, das exemplarische Lernen, das Fördern von Multiperspektivität, die analytische Auseinandersetzung mit Geschichts- und Erinnerungskultur und eine identitätsstiftende Funktion gehören (vgl. KÜHBERGER / NEUREITER 2017:21).

In diesem Sinne wurde das Thema Erinnerungskulturen in den neuen Lehrplan für das integrative Fach Geschichte und Sozialkunde / Politische Bildung der Sekundarstufe I aufgenommen (vgl. LEHRPLAN 2016). In diesem im Jahr 2015/2016 pilotierten und seit 2016/2017 schulstufenweise in Kraft getretenen Lehrplan ist der Lehrstoff modular und nicht vordergründig chronologisch gestaltet. ${ }^{5}$ Teil dieser Struktur ist das Modul 6 „Geschichtskulturen - Erinnerungskulturen - Erinnerungspolitik“, das für die 8. Schulstufe vorgesehen ist und das Zusammenwirken von historischem und politischem Lernen berücksichtigt. Dieses sieht vor, dass Schülerinnen und Schüler die Instrumentalisierung von Geschichte und somit die öffentliche Funktion von Erinnerungskulturen kritisch hinterfragen (vgl. HeLlmUTH 2016). Damit wird vor allem die Dekonstruktionskompetenz gefördert, aber auch die historische Orientierungs- oder politische Urteilskompetenz spielen eine zentrale Rolle.

Die Implementierung und konkrete Umsetzung eines neuen Lehrplans nimmt bekanntlich viel Zeit in Anspruch und ist letzten Endes vom Engagement der Lehrkräfte abhängig. Somit ist es für eine Evaluierung der Unterrichtspraxis leider noch zu früh, vor allem da der Lehrplan erst ab dem Schuljahr 2018/2019 in der 8. Schulstufe eingeführt wurde. Auch die Anpassung der Schulbücher braucht einen zeitlich angemessenen Vorlauf, ist aber inzwischen abgeschlossen. Inwieweit die Ansätze des Lehrplans in die neuen Lehrwerke Eingang gefunden haben, soll im Folgenden anhand eines ausgewählten Exemplars im Sinne einer ersten Annäherung untersucht werden.

\subsection{Erinnerungskulturen als Gegenstand von Schulbüchern der Sekundar- stufe I}

$\mathrm{Zu}$ diesem Zweck muss die zentrale Bedeutung des Schulbuchs für den Geschichtsunterricht berücksichtigt werden. Während einerseits im Zuge des digitalen Wandels

5 Im Kommentar zum Lehrplan heißt es dazu: „Diese Module sind als verpflichtend zu unterrichtende Bündelungen zu verstehen und daher geschlossen zu unterrichten. Allerdings kann die Reihung der Module, wie sie im Curriculum vorgenommen wird, aufgehoben werden“ (HELlMUTH / KÜHBERGER 2016). 
gerade für das Fach Geschichte der sogenannte Leitmedienwechsel und damit die Veränderung der Zugänge zu Wissen und Information betont werden, kommen neuere Schulbuchanalysen zu dem Ergebnis, dass das gedruckte Schulbuch ,nach wie vor als ,Leitmedium des Geschichtsunterrichts““ gelten kann, so z.B. Thünemann (THÜNEMANN 2018:17). Gleichzeitig zeigt Ina Markova, wie sich die „Veränderungen österreichischer Geschichtsbilder“ in den Schulbüchern zwischen 1957 und 2010 manifestiert haben (MARKOVA 2013:261). Insgesamt nimmt die Intensität von Analysen österreichischer Geschichtsschulbücher seit einigen Jahren zu. Dabei wird vor allem das Thema Holocaust in den Fokus gerückt (vgl. MitTNIK 2017; BILEWICZ 2016), während der allgemeine Gegenstandsbereich Erinnerungskulturen eher eine marginale Rolle spielt. Wirft man dazu beispielsweise einen Blick in das Lehrwerk Zeitfenster aus dem Jahr 2010, das im Verlag Ed. Hölzel erschienen ist, finden sich unter der Überschrift „Umgang mit der ,braunen' Vergangenheit“ lediglich drei Sätze mit einer knappen Information zur Waldheim-Affäre. Eine Anregung zur kritischen Reflexion von Erinnerungskulturen ist darin nicht enthalten (vgl. BEIER / LEONHARDT 2010:84f.).

Im Gegensatz dazu greifen die aktuellen Geschichtsschulbücher die Vorgaben des neuen Lehrplans auf und die Orientierung an der modularen Struktur ist bereits im Inhaltsverzeichnis erkennbar. Exemplarisch hervorzuheben ist das aktuelle Schulbuch Querdenken 4. Geschichte und Politische Bildung vom Österreichischen Bundesverlag Schulbuch (ÖBV) aus dem Jahr 2019. Das Modul 6 „Geschichtskulturen - Erinnerungskulturen - Erinnerungspolitik“ erstreckt sich einschließlich Übungsteil über insgesamt 14 Seiten (MADER / STRUTZ 2019:137-150), was bereits einen quantitativen Zugewinn bedeutet. Des Weiteren wird von Vornherein ein analytisch-kritischer Zugang gewählt. Es werden dementsprechend die Funktionen von Erinnern und Gedenken, die damit einhergehende Instrumentalisierung und Kommerzialisierung sowie die Auswirkungen auf das Geschichtsbewusstsein bereits im ersten Inputtext thematisiert. In diesem Zusammenhang werden didaktische Prinzipien wie Multiperspektivität, Konstruktivität und Gegenwartsund Zukunftsbezüge berücksichtigt. Dazu trägt vor allem das Kapitel „Erinnern und Gedenken im Wandel" bei, in dem die Opferthese als zentrales Narrativ der Nachkriegszeit kritisch eingeordnet wird und Schülerinnen und Schüler zum Nachdenken über die Bedingungen für einen veränderten Umgang mit der NS-Zeit in Österreich angeregt werden.

Dieser kleine Eindruck ersetzt selbstverständlich keine systematische Analyse, in die auch eine kritische Betrachtung der Arbeitsaufträge, der verwendeten Bilder und Quellenauszüge einbezogen werden müsste. Abzuzeichnen scheint sich jedoch, dass auf der Basis des neuen Lehrplans für die Sekundarstufe I dem Thema 
Erinnerungskulturen in den Geschichtsschulbüchern nicht nur mehr Raum eingeräumt wird, sondern die Art und Weise der Aufbereitung eine kritische Beschäftigung mit dem Gegenstand ermöglicht. Von Bedeutung ist das vor allem, weil viele Lehrerinnen und Lehrer in ihrem Unterricht strikt nach dem Schulbuch vorgehen und somit der Weg zur thematischen Umsetzung in der konkreten Unterrichtspraxis geebnet werden kann.

\subsection{Empirische Zugänge}

Neben den curricularen Vorgaben und der Entwicklung passender Schulbücher gehört zu einer reflektierten Arbeit mit Erinnerungskulturen im historisch-politischen Unterricht die Frage nach den Vorerfahrungen der Schülerinnen und Schüler. Einen ersten Einblick in Schülerinnen- und Schülervorstellungen zum Umgang mit Nationalsozialismus, Holocaust und Erinnerungskulturen gibt eine empirische Untersuchung aus geschichtsdidaktischer Perspektive, die an Salzburger Neuen Mittelschulen durchgeführt wurde (vgl. KÜHBERGER/NEUREITER 2017).

Anlass für die Befragung bildete eine Reihe von Schmierereien und Beschädigungen von Stolpersteinen zum Gedenken an NS-Opfer oder Denkmälern wie das Euthanasie-Denkmal bei Schloss Mirabell in Salzburg in den Jahren 2013 und 2014. Hauptziel der Studie war somit herauszufinden, ,inwieweit und wie Denk- bzw. Mahnmäler für die Opfer des Nationalsozialismus Eingang in das schulische historische und historisch-politische Lernen finden" (KÜHBERGER / NEUREITER 2017:22). Durchgeführt wurde die quantitative Studie im Sommersemester 2015 in der 8. Schulstufe und es nahmen insgesamt 283 Schülerinnen und Schüler im Alter von 13 bis 17 Jahren teil. Ergänzend dazu hat im Wintersemester 2015/2016 eine Befragung von 25 Lehrkräften im Alter von 29 bis 62 Jahren stattgefunden (vgl. KÜHBERGER / NEUREITER 2017:36f.).

Aus der Vielzahl an Ergebnissen können nur einige wesentliche Aspekte herausgegriffen werden. Interessant ist zunächst, dass die Schülerinnen und Schüler ihr Wissen über den Nationalsozialismus eher als durchschnittlich bewerten und äquivalent dazu auch Defizite beim Basiswissen zum Themenbereich zeigen. Auffällig groß ist in diesem Zusammenhang die Zustimmung zur Opferthese. Gleichzeitig hat der Unterricht für die Wissensaneignung aus Schülerinnen- und Schülerperspektive einen zentralen Stellenwert (vgl. KÜHBERGER / NEUREITER 2017:44f.). Dabei ist ein Überdruss an der Beschäftigung mit Nationalsozialismus und Holocaust im Geschichtsunterricht nicht erkennbar (vgl. KÜHBERGER / NEUREITER 2017:58f.).

Insgesamt stehen die Schülerinnen und Schüler der Auseinandersetzung mit der NS-Vergangenheit also aufgeschlossen gegenüber und über die Hälfte der 
Befragten lehnt die Behauptung ab, dass der Nationalsozialismus für sie keine Bedeutung hätte. Auch der Notwendigkeit einer vielfältigen Gedenkkultur für die Opfer des Nationalsozialismus stimmt ein Großteil der Schülerinnen und Schüler weitgehend zu (vgl. KÜHBERGER / NEUREITER 2017:65f.).

Parallel dazu ist das Interesse der Lehrerinnen und Lehrer an den Themen Nationalsozialismus und Holocaust groß und wird auch auf Seite der Schülerinnen und Schüler als hoch eingeschätzt (vgl. KÜHBERGER / NEUREITER 2017:86). Während der Stellenwert der offiziellen Erinnerungskulturen in Österreich als gering bewertet wird, sprechen sich weit über die Hälfte der befragten Lehrkräfte dafür aus, „im Unterricht auch den Umgang mit der österreichischen Erinnerungskultur (Museen, Gedenkstätten etc.) umfassend zu beleuchten“ (KÜHBERGER / NEUREITER 2017:88f.). Zum Zeitpunkt der Befragung befand sich der aktuelle Lehrplan für Geschichte und Sozialkunde / Politische Bildung an der Sekundarstufe I noch in der Pilotierung, sodass die teilnehmenden Lehrerinnen und Lehrer mit dem für die 8. Schulstufe entwickelten Modul 6 noch nicht vertraut waren. Eine erneute bzw. ergänzende Befragung zum Themenkomplex Erinnerungskulturen nach der Einführung des Moduls im Schuljahr 2018/2019 könnte Aufschluss über die Haltung zu den curricularen Vorgaben geben.

\section{Fazit}

Zusammenfassend lässt sich sagen, dass das Thema ,Erinnerungskulturen“ in Österreich mit dem neuen Lehrplan für die Sekundarstufe I im Sinne eines historisch-politischen Zugangs gestärkt wurde, was in einem eigenen Modul zum Ausdruck kommt. Diesem Stellenwert tragen auch die aktualisierten Schulbücher Rechnung. Des Weiteren zeigen empirische Ergebnisse aus Salzburg, dass sowohl auf Seiten der Schülerinnen und Schüler als auch der Lehrerinnen und Lehrer Interesse an dem Thema besteht. Da die Untersuchung für Österreich nicht als repräsentativ gelten kann, wären weiterführende, auch qualitative Studien notwendig, um diesen Befund zu überprüfen.

Während also einerseits der neue Lehrplan für die Sekundarstufe I Rahmenbedingungen für eine kritisch-reflektierte Auseinandersetzung mit Erinnerung und Gedenken an den Nationalsozialismus schafft und andererseits die Aufgeschlossenheit bei Schülerinnen und Schülern sowie Lehrkräften gegenüber diesem Gegenstandsbereich groß ist, braucht es entsprechende Lernräume für die Um- 
setzung im Unterricht. Hier ist letzten Endes auch die Lehrerinnen- und Lehrerausbildung gefragt, einen Beitrag zur Bewusstseinsbildung für die gesellschaftspolitische und didaktische Bedeutung des Themas zu leisten.

\section{Literatur}

Assmann, Aleida (2005): Jahrestage - Denkmäler in der Zeit. In: MÜNCH, PAUl (ed.): Jubiläum, Jubiläum .... Zur Geschichte öffentlicher und privater Erinnerung. Essen, 305-314. Assmann, Aleida (2013): Das neue Unbehagen an der Erinnerungskultur. Eine Intervention. München.

BEIER, ROBERT / LEONHARDT, Ute (2010): Zeitfenster 4. Wien.

Bergmann, Klaus (2009): Gedenktage, Gedenkjahre und historische Vernunft. In: Horn, SABINe / SAUER, Michael (eds.): Geschichte und Öffentlichkeit. Orte - Medien - Institutionen. Göttingen, 24-31.

Bilewicz, Wolfgang (2016): Der Holocaust in Schulbüchern und Lehrplänen. Ein historisch-pädagogischer Vergleich zwischen Bayern und Österreich. Marburg.

BRÜNING, CHRISTINA IsABEL (2018): Holocaust Education in der heterogenen Gesellschaft. Eine Studie zum Einsatz videographierter Zeugnisse von Überlebenden der nationalsozialistischen Genozide im Unterricht. Frankfurt a.M.

CORNELIßEN, CHRISTOPH (2012): Erinnerungskulturen. Version: 2.0: http://docupedia.de/ zg/cornelissen_erinnerungskulturen_v2_de_2012 (09.10.2019).

Cornelißen, Christoph / Klinkhammer, Lutz / SchWentKer, Wolfgang (2003): Nationale Erinnerungskulturen seit 1945 im Vergleich. In: CORNELIßEN, CHRISTOPH / KLINKHAMmer, LutZ / SchweNTKER, WolfGANG (eds.): Erinnerungskulturen. Deutschland, Italien und Japan seit 1945. Frankfurt a.M., 9-27.

Demantowsky, Marko (2015): ,,Public History“-Aufhebung einer deutschsprachigen Debatte? In: Public History Weekly 3, 2: https://public-history-weekly.degruyter.com/ 3-2015-2/public-history-sublation-german-debate/ (09.10.2019).

ERLL, ASTRID (2008): Kollektives Gedächtnis und Erinnerungskulturen. In: NÜNNING, ANSGAR/NÜNNING, VERA (eds.): Einführung in die Kulturwissenschaften. Theoretische Grundlagen - Ansätze - Perspektiven. Stuttgart, 156-185.

Gerald, John (2018): Österreich gedenkt des „Anschlusses“. In: Der Standard v. 12.03.2018: https://derstandard.at/2000075903699/Oesterreich-gedenkt-des-Jahrestags-des-Anschlusses (30.09.2019).

HAMMERSTEIN, KATRIN (2017): Gemeinsame Vergangenheit - getrennte Erinnerung? Der Nationalsozialismus in Gedächtnisdiskursen und Identitätskonstruktionen von Bundesrepublik Deutschland, DDR und Österreich. Göttingen.

HASBERG, WOLFGANG (2004): Erinnerungskultur - Geschichtskultur, Kulturelles Gedächtnis - Geschichtsbewusstsein. 10 Aphorismen zu begrifflichen Problemfeldern. In: Zeitschrift für Geschichtsdidaktik 3:198-207. 
Hellmuth, Thomas (2016): Erinnern und vergessen. Erinnerungskultur im neuen Lehrplan der Sekundarstufe I. In: Historische Sozialkunde 3:11-15.

Hellmuth, Thomas (2018): Späte Einsicht, intensives Erinnern. Österreich heute. In: Public History Weekly 6, 38: https://public-history-weekly.degruyter.com/6-2018-38/naziremembrance-austria-today/ (09.10.2019).

Hellmuth, ThOMAs / KÜHBERGER, CHRISTOPH (2016): Kommentar zum Lehrplan der Neuen Mittelschule und der AHS-Unterstufe ,,Geschichte und Sozialkunde / Politische Bildung “: https://www.politik-lernen.at/dl/NqssJKJKonmomJqx4OJK/GSKPB_Sek_I_2016 _Kommentar_zum_Lehrplan_Stand_26_09_2016.pdf (09.10.2019).

Homepage zum Gedenk- und Erinnerungsjahr 2018. https://www.oesterreich100.at/ events-und-publikationen.html (30.09.2019)

Knigge, Volkhard (2010): Zur Zukunft der Erinnerung. In: Aus Politik und Zeitgeschichte 25-26:10-16.

KNIGHT, RoBert (2006): Einige Gedanken zum Gedenkjahr. In: KrAMER, HeLmUt / LIEBHART, KARIN / STADLER, FRIEDRICH (eds.): Österreichische Nation - Kultur - Exil und Widerstand. In memoriam Felix Kreissler. Wien, 297-302.

KRAMMER, REINHARD (2003): Anmerkungen zu einer Didaktik des Erinnerns in Österreich. In: Historische Sozialkunde 4:14-16.

KRAMMER, REINHARD (2004): Zeitgeschichte in der Schule - ein didaktischer Problemaufriss. In: Informationen zur Politischen Bildung 20:1-9.

KÜHBERGER, CHRISTOPH (2010): Erinnerungskulturen als Teil des historisch-politischen Lernens. In: Informationen zur Politischen Bildung 32:39-42.

KüHBERger, Christoph / NeureIter, Herbert (2017): Zum Umgang mit Nationalsozialismus, Holocaust und Erinnerungskultur. Eine quantitative Untersuchung bei Lernenden und Lehrenden an Salzburger Schulen aus geschichtsdidaktischer Perspektive. Schwalbach/Ts.

Lehrplan Geschichte und Sozialkunde / Politische Bildung Sek. I (2016). https://www.politiklernen.at/dl/mnoNJKJKonmomJqx41JK/Gesetzesblatt_113._Verordnung_18_Mai_2016.pdf (09.10.2019).

Mader, Sabine / Strutz, Andrea (2019): Querdenken 4. Geschichte und Politische Bildung. Wien.

Markova, InA (2013): Wie Vergangenheit erzählt wird. Der Umgang mit der NS-Zeit in österreichischen Schulbüchern. Marburg.

MitTNIK, PHILIPP (2017): Holocaust-Darstellungen in Schulbüchern. Deutsche, österreichische und englische Lehrwerke im Vergleich. Schwalbach/Ts.

MitTNIK, PhILIPP (2018): Nationalsozialismus im Schulbuch. Geschichtsdidaktische Zugänge in deutschen, österreichischen und englischen Lehrwerken der Sekundarstufe I. In: Bramann, Christoph / KüHBerger, Christoph / Bernhard, Roland (eds.): Historisch Denken Lernen mit Schulbüchern. Frankfurt a.M., 111-135. 
TAUBITZ, JAN (2016): Holocaust Oral History und das lange Ende der Zeitzeugenschaft. Göttingen.

ThÜNemann, Holger (2018): Historisch denken lernen mit Schulbüchern? Forschungsstand und Forschungsperspektiven. In: BramanN, CHristoPH / KüHBERGER, CHRISTOPH / BERNHARD, Roland (eds.): Historisch Denken Lernen mit Schulbüchern. Frankfurt a.M., 17-62.

Uhl, HeIDEMARIE (2010): Warum Gesellschaften sich erinnern. In: Informationen zur Politischen Bildung 32:5-14.

WelskopP, THOMAs (2014): Jubiläen und Gedenktage als Anker der öffentlichen Erinnerungskultur. Vortrag auf der Herbsttagung der Fachgruppe Geschichtsmuseen im Deutschen Museumsbund vom 15. bis 17. November 2014 in Bielefeld: https://www.museumsbund.de /wp-content/uploads/2018/05/2014-2-welskopp-gedenktage.pdf (16.09.2019).

WOLFrum, EdGar (2001 / 52011): Geschichtspolitik. In: NOHLEN, DieTER/ Grotz, Florian (eds.): Kleines Lexikon der Politik. München, 207-210. 